KOMPASS

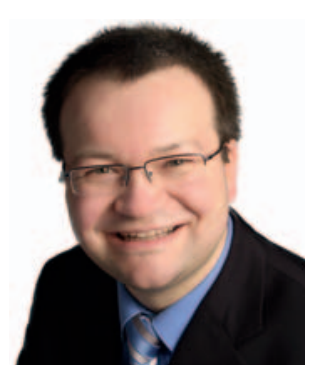

Marek Lommatzsch

Zentrum für Innere Medizin,

Universität Rostock

\title{
Allergische Atemwegserkrankungen im Spannungsfeld zwischen Versorgung und wissenschaftlichem Fortschritt
}

Der Schwerpunkt der aktuellen Ausgabe von Karger Kompass Pneumologie beschäftigt sich mit dem Thema «Allergische Atemwegserkrankungen». Dieses Thema ist sehr aktuell und von großer Relevanz im klinischen Alltag. Die Prävalenz der Rhinitis liegt in Deutschland bei 15\%, die Asthma-Prävalenz bei 5-10\% [1]. Allergien sind häufigster Auslöser einer chronischen Rhinitis und/oder eines Asthma und gehören somit zu den großen Volkserkrankungen. Glücklicherweise beobachten wir große aktuelle Fortschritte in der Prävention, Diagnostik und Therapie von allergischen Atemwegserkrankungen. Ein Beispiel ist die spezifische Allergen-Immuntherapie, welche sich 1. hinsichtlich der verschiedenen Applikationswege (subkutan, sublingual, oral oder intralymphatisch) [2], 2. der verschiedenen Indikationen bei allergischer Rhinitis und allergischem Asthma [3] und 3. der Qualitätsstandards der Präparate und Zulassungen [4] in den letzten Jahren beeindruckend entwickelt hat. Leider sind die Betroffenen in Deutschland trotz hoher Prävalenz und trotz der Fortschritte in der Prävention, Diagnostik und Therapie immer noch erschreckend unterversorgt [5]. Hierfür gibt es Gründe aufseiten der Ärzteschaft und der Patienten [6]:

- Ärzte: Atemwegserkrankungen wie Rhinitis und/oder Asthma werden oft nur als Organerkrankungen mit einer
Basistherapie zügig behandelt, eine allergologische Abklärung oder eine Überweisung zum allergologisch geschulten Facharzt bleibt häufig aus. Zudem verfügen auch Fachärzte der einzelnen Disziplinen oft nicht über die Zusatzbezeichnung «Allergologie» oder haben kein Interesse an der Allergologie. Dies wird dadurch verstärkt, dass die Vergütung allergologischer Diagnostik und Therapie in Deutschland im Vergleich zu anderen «lukrativeren» Prozeduren schlecht ist.

- Patienten: Das Bewusstsein für die Art, die große Bedeutung und die vielen Therapieoptionen allergologischer Erkrankungen ist in der deutschen Bevölkerung gering ausgeprägt. Trotz typischer und heftiger Beschwerden erfolgt oft kein Arztbesuch. Die Bagatellisierung von Allergien wird dadurch noch befördert, dass einige antiallergische Medikamente (wie Antihistaminika) nicht verschreibungspflichtig sind.

Somit erleben wir in Deutschland das seltsame Paradoxon einer erheblichen Unterversorgung von Patienten mit allergischen Atemwegserkrankungen trotz eines sehr gut finanzierten Gesundheitssystems und trotz großer Fortschritte in der Therapie dieser Erkrankungen [6]. Dieser Diskrepanz zwischen abnehmender Versorgung einerseits und zunehmenden Therapieoptionen andererseits muss durch gute Aufklärung und Fortbildung dringend entgegengewirkt werden. Das vorliegende Heft von Karger Kompass PNEUMOLOGIE kommt daher mit seinem thematischen Schwerpunkt zur rechten Zeit. Einen zentralen Aspekt bei der Diagnostik und Therapie allergischer Atemwegserkrankungen stellt die genaue Erfassung der Allergene dar, welche Atemwegserkrankungen auslösen. Hierbei spielen «Allergendatenbanken» im Internet eine immer größere Rolle. Prof. Christian Radauer von der Medizinischen Universität Wien vergleicht in seinem sehr lesenswerten Artikel [7] die verschiedenen verfügbaren Datenbanken und gibt dem Leser wertvolle Tipps für die Orientierung im «Allergendschungel». Prof. Christian Vogelberg vom Universitäts-AllergieCentrum Dresden geht in seinem Wissenstransfer [8] auf die spannende Wechselbeziehung zwischen allergischen Atemwegserkrankungen und Hauterkrankungen ein und zeigt das breite Spektrum allergologischer Fragestellungen auf. Besonders lesenswert ist auch das Interview mit Frau Dr. Braun [9], der Trägerin des europäischen Allergieforschungspreises 2017 über ihre aktuellen Forschungen und die Perspektiven der Allergieforschung in Europa.

Neben dem allergologischen Schwerpunkt hält dieses Heft aber noch weitere pneumologische «Leckerbissen» für den Leser bereit: von der unkonventionellen

\section{KARGER}

Fax +497614520714 information@karger.com www.karger.com (c) 2017 S. Karger GmbH, Freiburg
Prof. Dr. Marek Lommatzsch

Zentrum für Innere Medizin

Universität Rostock

Ernst-Heydemann-Straße 6, 18057 Rostock, Deutschland

marek.lommatzsch@med.uni-rostock.de 
Anwendung von Endobronchial-Ventilen bei Tuberkulose [10] (welche wie ein Nachhall aus der Geschichte der Tuberkulosetherapie wirkt) über die verschiedenen Leitlinien zur Sauerstofftherapie [11] und aktuelle Diskussionen zur Bedeutung der Kryobiopsie [12] bis hin zu den Nebenwirkungen der sich rasant entwickelnden Tumor-Immuntherapie [13].

Somit spannt dieses gelungene Heft den großen pneumologischen Bogen von den häufigen allergischen Erkrankungen zu den häufigen nicht-allergischen Erkrankungen der Lunge, und

\section{Literatur}

1 Langen U, Schmitz R, Steppuhn H: Häufigkeit allergischer Erkrankungen in Deutschland. Bundesgesundheitsbl 2013;56:698-706.

2 Klimek L, Pfaar O, Bousquet J, Senti G, Kundig T: Allergen immunotherapy in allergic rhinitis: current use and future trends. Expert Rev Clin Immunol 2017;15:1-10.

3 Virchow J, Backer V, Kuna P, et al.: Efficacy of a house dust mite sublingual allergen immunotherapy tablet in adults with allergic asthma: a randomized clinical trial. JAMA 2016;315:1715-1725.

4 Pfaar O, Bachert C, Bufe A, et al.: Leitlinie zur (allergen-)spezifischen Immuntherapie bei IgE-vermittelten allergischen Erkrankungen. Allergo J Int 2014;23:282.

5 Biermann J, Merk HF, Wehrmann W, Klimek L, Wasem J: Allergische Erkrankungen der Atemwege - Ergebnisse einer umfassenden Patientenkohorte in der deutschen gesetzlichen Krankenversicherung. Allergo J 2013;22:366-373.

6 Lommatzsch M, Koschel D, Welte T: Allergologische Versorgung in Deutschland: ein Paradoxon. Pneumologie 2013;67:659-661.

7 Radauer C: Wegweiser durch den Allergendschungel: Allergendatenbanken, ihre Merkmale und Anwendungsgebiete. Karger Kompass Pneumol 2017:5:137-148. gibt dem Leser faszinierende Einblicke in unser Fachgebiet. Ich wünsche Ihnen viel Freude und Erkenntnisgewinn bei der Lektüre!

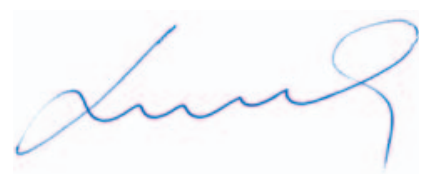

Prof. Dr. Marek Lommatzsch

8 Vogelberg C: Chronische spontante Urtikaria bei Asthmatikern: Das Rätsel um reziproke Mechanismen. Karger Kompass Pneumol 2017; 5:149-150.

9 Braun A: Acht Fragen an die Trägerin des ADF/ECARF-Award for European Allergy Research 2017. Karger Kompass Pneumol 2017;5:176-177.

10 Krüger S: Endobronchiale Ventil-Implantation bei multiresistenter Tuberkulose: Erfolg versprechende Methode bei negative Kollateralventilation. Karger Kompass Pneumol 2017;5:152-154.

11 Haidl P: Vergleich der deutschen und britischen Leitlinien zur LangzeitSauerstofftherapie: Grundlagen zu Verordnungskriterien für LTOT bleiben ungeklärt. Karger Kompass Pneumol 2017;5:166-167.

12 Wagner M: Erfolgreiche Lungen-Kryobiopsie bei diffuser Lungenparenchymerkrankung: Strenge Ein- und Ausschlusskriterien, exakte Bildgebung und erfahrenes Komplikationsmanagement sind essenziell. Karger Kompass Pneumol 2017;5:156-158.

13 Imafuku K, Yoshino K, Yamaguchi K, et al.: Zwei Fälle einer wiederholten Anwendung von Nivolumab nach einer Pneumonitis als immunvermittelte Nebenwirkung. Karger Kompass Pneumol 2017;5:185-188. 


\section{FOKUS}

\section{Allergische Atemwegserkrankungen}

\section{Epidemiologie}

Immer mehr Menschen leiden unter Allergien - etwa gegen Blütenpollen oder Nahrungs- und Arzneimittel: Allein in Deutschland ist etwa jeder Dritte davon betroffen. Frauen sind generell häufiger betroffen als Männer und Jüngere häufiger als Ältere. Die drei häufigsten allergischen Krankheitsbilder umfassen Heuschnupfen, allergisches Asthma und Neurodermitis und haben eines gemeinsam: Es handelt sich um die Folge einer Überreaktion des Immunsystems, das auf eigentlich harmlose Substanzen aus der Umwelt wie Gräserund Blütenpollen, Hausstaubmilben oder Tierhaare mit krankmachenden Abwehrreaktionen reagiert. Der Grund dafür ist, dass bestimmte Eiweiße oder eiweißartige Strukturen in den Nahrungsmitteln und Blütenstäuben (fast) identisch sind. Dies kann auch zu Kreuzallergien führen.

\section{Herausforderungen und Handlungsbedarf}

Als Ursache für die drastische Zunahme von Allergien diskutieren Forscher verschiedene Faktoren, darunter mögliche Wirkungen von Schadstoffen in der Umwelt [1] oder etwa die Frage, ob das kindliche Immunsystem heute weniger als früher trainiert wird. Seit einigen Jahren richten Allergologen ihr Augenmerk verstärkt auf eine in Deutschland eigentlich gebietsfremde Pflanze, die Beifuß-Ambrosie, deren Pollen stark Allergie auslösend sind. Betroffene Organe bei Allergikern sind zumeist Atemwege oder Haut, häufig beide zusammen.

Viele Fragen bleiben bislang offen, z.B. was macht einen Stoff überhaupt zum Allergen, warum bestehen zwischen den verschiedenen Allergenen so große Unterschiede in ihrem jeweiligen allergenen Potenzial, welche besondere Rolle spielen Verkehrsemissionen für das Allergiegeschehen tatsächlich etc. Auch die immunologischen Mechanismen, welche der Toleranzentwicklung bei der spezifischen Immuntherapie (SIT) oder Hyposensibilisierung zugrunde liegen, sind ebenfalls bis heute nicht vollständig verstanden - genauso die Bedeutung von Genen auf die Entwicklung von Allergien.

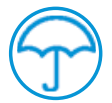

\section{Prävention}

In der gemeinsam von den führenden Fachgesellschaften verabschiedeten aktualisierten Leitlinie zur Allergieprävention [2] sind einige Maßnahmen im Wesentlichen in Hinblick auf Lebensgewohnheiten und Ernährung zusammengestellt, die zum Schutz vor Allergien empfohlen werden.

Neben einer Stillzeit von 4 Monaten, der Vermeidung felltragender Haustiere bei Risikokindern sowie Übergewicht beschäftigen sich zwei neue Aspekte der Leitlinie mit psychosozialen Faktoren und der Geburt von Babys. Im Vergleich zur normalen Geburt birgt ein Kaiserschnitt ein höheres Allergierisiko für das Kind. Psychosoziale Faktoren wie z.B. die Trennung der Eltern oder der Tod eines nahen Verwandten während der Schwangerschaft oder in der frühen Kindheit, werden ebenfalls mit einem erhöhten Allergierisiko des Babys in Verbindung gebracht. Zudem zeigen Kinder, die auf einem traditionellen Bauernhof bzw. in traditioneller Landwirtschaft aufwachsen, ein deutlich geringeres Risiko für Asthma [3]. Diesbezüglich wird derzeit auch untersucht, inwieweit eine spezifische Zusammensetzung des «Mikrobioms» einen Schutz vor Asthma und Allergien bietet [4]. Auch der Besuch einer Kindertagesstätte in den ersten zwei Lebensjahren oder eine höhere Anzahl älterer Geschwister haben Asthma-präventive Wirkungen.

\section{Therapieoptionen}

Für die Behandlung von Heuschnupfen und Asthma stehen diverse Optionen zur Verfügung. Die topischen nasalen (allergische Rhinitis) und die inhalativen Steroide (Asthma) stellen die Basistherapie der Erkrankungen dar, auch bei Kindern und Schwangeren. Orale Steroide sind hingegen möglichst aufgrund der Nebenwirkungen in der Dauertherapie zu vermeiden. Antihistaminika haben eine große Bedeutung bei der Behandlung der allergischen Rhinitis, beim allergischen Asthma spielen sie eine geringere Rolle. Die Wirkung von Cromonen, darunter Nedocromil, ist lediglich bei lokaler Anwendung als Nasenspray oder Augentropfen nachgewiesen. Große Fortschritte in der Behandlung von Rhinitis aber auch der Prävention und Therapie von allergischem Asthma sind im Bereich der SIT zu verzeichnen: Neue Applikationswege (subkutan, sublingual, oral oder intralymphatisch) [5], neue Indikationen bei allergischer Rhinitis und allergischem Asthma [6] und bessere Qualitätsstandards der Präparate und Zulassungen [7] haben zu einer zunehmenden klinischen Bedeutung der SIT beigetragen. Gerade auch bei Patienten mit allergischem Asthma kommt die SIT zunehmend zum Einsatz. Bei Patienten mit schweren Formen des allergischen Asthmas, bei denen die SIT vor allem aus Sicherheitsgründen kontraindiziert ist, kann gegebenenfalls eine kombinierte Therapie von SIT mit Biologika (wie dem monoklonalen Antikörper Omalizumab) zum Einsatz kommen.

\section{Literatur}

1 Beck I, Jochner S, Gilles S, et al.: High environmental ozone levels lead to enhanced allergenicity of birch pollen. PLoS One 2013;20:e80147.

2 AWMF: Leitlinie Allergieprävention: http://www.awmf.org/leitlinien/detail/ I//061-016.htm/ (letzter Zugriff 09.08.2017)

3 Stein MM, Hrusch CL, Gozdz J, et al.: Innate immunity and asthma risk in amish and hutterite farm children. N Engl J Med 2016;375:411-421.

4 von Mutius E: The microbial environment and its influence on asthma prevention in early life. J Allergy Clin Immunol 2016;137:680-689.

5 Klimek L, Pfaar O, Bousquet J, et al.: Allergen immunotherapy in allergic rhinitis: current use and future trends. Expert Rev Clin Immunol 2017;15:1-10.

6 Virchow J, Backer V, Kuna P, et al.: Efficacy of a house dust mite sublingual allergen immunotherapy tablet in adults with allergic asthma: a randomized clinical trial. JAMA 2016;315:1715-1725.

7 Pfaar O, Bachert C, Bufe A, et al.: Leitlinie zur (allergen-)spezifischen Immuntherapie bei IgE-vermittelten allergischen Erkrankungen. Allergo J Int 2014;23:282.

Prof. Dr. Marek Lommatzsch, Zentrum für Innere Medizin, Universität Rostock 